\title{
Drug interaction prediction using ontology-driven hypothetical assertion framework for pathway generation followed by numerical simulation
}

\author{
Takeshi Arikumaํ, Sumi Yoshikawa², Ryuzo Azuma², Kentaro Watanabe ${ }^{1}$, \\ Kazumi Matsumura ${ }^{2}$ and Akihiko Konagaya*1,2
}

Address: ${ }^{1}$ Department of Computer Science, Tokyo Institute of Technology, 2-12-1 Oookayama, Meguro, Tokyo, Japan and ${ }^{2}$ Genomic Sciences Center, RIKEN, 1-7-22 Suehiro, Tsurumi, Yokohama, Kanagawa, Japan

Email: Takeshi Arikuma - atake@bio.cs.titech.ac.jp; Sumi Yoshikawa - sumi@bio.cs.titech.ac.jp; Ryuzo Azuma - azuma@bio.cs.titech.ac.jp; Kentaro Watanabe - kentaro.watanabe.dc@hitachi.com; Kazumi Matsumura - matsumura-k@admet.daiichichem.co.jp;

Akihiko Konagaya* - kona@bio.cs.titech.ac.jp

* Corresponding author

from Symposium of Computations in Bioinformatics and Bioscience (SCBB07)

lowa City, lowa, USA. 13-15 August 2007

Published: 28 May 2008

BMC Bioinformatics 2008, 9(Suppl 6):SI I doi:10.1 I86/I47I-2105-9-S6-SI I

This article is available from: http://www.biomedcentral.com/I47I-2I05/9/S6/SII

(C) 2008 Arikuma et al; licensee BioMed Central Ltd.

This is an open access article distributed under the terms of the Creative Commons Attribution License (http://creativecommons.org/licenses/by/2.0), which permits unrestricted use, distribution, and reproduction in any medium, provided the original work is properly cited.

\begin{abstract}
Background: In accordance with the increasing amount of information concerning individual differences in drug response and molecular interaction, the role of in silico prediction of drug interaction on the pathway level is becoming more and more important. However, in view of the interferences for the identification of new drug interactions, most conventional information models of a biological pathway would have limitations. As a reflection of real world biological events triggered by a stimulus, it is important to facilitate the incorporation of known molecular events for inferring (unknown) possible pathways and hypothetic drug interactions. Here, we propose a new Ontology-Driven Hypothetic Assertion (OHA) framework including pathway generation, drug interaction detection, simulation model generation, numerical simulation, and hypothetic assertion. Potential drug interactions are detected from drug metabolic pathways dynamically generated by molecular events triggered after the administration of certain drugs. Numerical simulation enables to estimate the degree of side effects caused by the predicted drug interactions. New hypothetic assertions of the potential drug interactions and simulation are deduced from the Drug Interaction Ontology (DIO) written in Web Ontology Language (OWL).

Results: The concept of the Ontology-Driven Hypothetic Assertion (OHA) framework was demonstrated with known interactions between irinotecan (CPT-II) and ketoconazole. Four drug interactions that involved cytochrome $\mathrm{p} 450$ (CYP3A4) and albumin as potential drug interaction proteins were automatically detected from Drug Interaction Ontology (DIO). The effect of the two interactions involving CYP3A4 were quantitatively evaluated with numerical simulation. The co-administration of ketoconazole may increase AUC and Cmax of SN38 (active metabolite of irinotecan) to $108 \%$ and $105 \%$, respectively. We also estimates the potential effects of genetic variations: the AUC and Cmax of SN-38 may increase to $208 \%$ and $165 \%$ respectively with the genetic variation UGTIAI*28/*28 which reduces the expression of UGTIAI down to $30 \%$.
\end{abstract}




\begin{abstract}
Conclusion: These results demonstrate that the Ontology-Driven Hypothetic Assertion framework is a promising approach for in silico prediction of drug interactions. The following future researches for the in silico prediction of individual differences in the response to the drug and drug interactions after the administration of multiple drugs: expansion of the Drug Interaction Ontology for other drugs, and incorporation of virtual population model for genetic variation analysis, as well as refinement of the pathway generation rules, the drug interaction detection rules, and the numerical simulation models.
\end{abstract}

\section{Background}

The role of in silico prediction of drug interactions on the pathway level is becoming more and more important for solving real-world problems. Multiple-drug regimens exemplify the need for the computer-assisted prediction of drug interactions. Multiple-drug regimens are commonly prescribed for elderly patients suffering from more than one disease. However, they sometimes cause unexpected severe side effects because of the drug interactions or individual differences concerning response to the drugs [1]. Therefore, the prediction of drug interactions for preventing the side effects is an important issue for these regimens.

On the other hand, information useful for in silico drug interaction prediction has increased very rapidly in recent years. Technological innovations in genomic sciences have produced an enormous amount of biomolecular information including sequences, structures, and pathways. In order to integrate the biomolecular information, ontology is attracting a lot of attention [2,3]. In addition, pharmacokinetics modeling and simulation are emerging, promising techniques to understand the dynamic behavior of drug metabolic pathways [4,5].

To develop a practical in silico drug interaction prediction system by integrating the above information and techniques, the following issues must be solved.

\section{Context dependency of drug-metabolic pathways}

Drug-metabolic pathways do not exist a priori. They strongly depend on contexts and situations including the administration route, single nucleotide polymorphism (SNP) of drug-response genes, and the administration of multiple drugs and foods. Therefore, a dynamic reconstruction of drug metabolic pathways from primitive molecular events is necessary for drug interaction prediction on the pathway level. Such reconstruction requires the formal definition of molecular events and the relations among them, i.e., the Drug Interaction Ontology (DIO).

\section{Treatment of multiscale events}

Pathways triggered by drug administration consist of multiscale events: from the molecular level to the body level, ranging from nanoseconds to hours or days in terms of drug response. For example, drug administration and drug excretion are body-level events, while drug transport and enzymatic reactions are molecular-level events. A comprehensive view from the molecular level to the body level is necessary in order to understand multi-scale events.

\section{Quantitative evaluation of interactions}

Quantitative evaluation plays an essential role to estimate the degree of side effects caused by drug interactions. More than one drug interactions may occur in drug-metabolic pathways from the qualitative reasoning view point. However, not all drug interactions cause side effects because of differences in binding affinity and molecular population. Quantitative simulation models with an in silico drug interaction prediction system must be incorporated to discriminate serious drug interactions from negligible ones. It should be also noted that total drug metabolism depends on not only kinetic parameters but also physiological parameters such as organ volumes and blood flows. Incorporation of kinetic parameters and physiological parameters is necessary for a realistic simulation model to recapture experimental data.

In this paper, we propose a new drug-interaction prediction framework called "Ontology-Driven Hypothetic Assertion (OHA)" focusing mainly on automatic generation of drug-metabolic pathways, automatic detection of drug interactions on multiple-drug regimens [6], and quantitative evaluation of the drug interactions with numerical simulation. The effectiveness of the OHA framework was demonstrated in the prediction of interactions between irinotecan (CPT-11) and ketoconazole.

\section{Design philosophy}

Ontology-Driven Hypothetical Assertion

The Ontology-Driven Hypothetical Assertion framework maps inferences onto the Drug Interaction Ontology as both machine and human understandable form. The inferences include the results of qualitative reasoning and numerical simulation, i.e. generated pathways and detected drug interactions and generated differential equations and simulation results. The assertions enable to interpret the obtained results such as drug interaction candidates, simulation models, the pharmacokinetic 
moment values with background knowledge on pharmaceutical science and biochemistry in the ontology.

\section{Causality-based pathway modularization}

Modularization is necessary for the dynamic reconstruction of pathways that depend on dose conditions. Careful selection of primitive modules is the key to ensuring the soundness of pathway reconstruction. Molecular events, such as molecular transport and enzymatic reactions, are well-formed primitive modules for this purpose. In this paper, we refer to the primitive modules as "molecular events", and the aggregation of molecular events as "pathways".

To avoid redundant pathway branch constructions, which are non-essential for the target drug interactions, we adopt causality-based modularization in which each molecular event is defined by the unique relationship between key molecules before and after the event. The triadic relationship <trigger, situator, resultant>, proposed by Yoshikawa et al. [7], is one such causality that can be commonly found in molecular reactions. For example, in case of enzymatic reactions, substrates, enzymes and other products correspond to trigger, situator and resultant, respectively. In the case of molecular transport, extra (intra) cellular molecules, transporters and intra (extra) cellular molecules correspond to the participants of the triadic relation, respectively. The triadic relationship can be applicable to higher level events like drug dosage and drug excretion, as long as its causality is unique and clear.
Figure $1(a)(b)(c)$ shows a simple example of pathway reconstruction with two primitive molecular events: an enzymatic reaction in which carboxylesterase (CE) metabolizes irinotecan into SN-38 (7-ethyl-10-hydroxycamptothecin) in the liver, and molecular transport in which $\mathrm{SN}-38$ in the liver is transported to the bile by MRP2 (Multidrug resistance-associated protein 2). Two molecular events are connected at the resultant of the enzymatic reaction (TR0000019) and the trigger of the molecular transport (TR0000007) for passing SN-38 in the liver (SN$38 @$ liver) to the bile.

\section{Ontology-based knowledge base construction}

The ontological approach in knowledge base design is adopted for resource sharing and the semantic description of molecular events and pathways. Ontology is necessary to define molecular events and pathways in a form that can be shared among computers and human beings. This enables the full use of powerful computational intelligence for dynamic pathway reconstruction in a way that human intelligence can follow and understand. Ontology is also important for establishing interoperability among web resources and thereby to make use of the latest drug reaction information published in the semantic web $[2,8]$. Public biological ontologies, especially in the field of chemical biology, are now dramatically increasing, and have a great potential to develop sustainable knowledge bases for molecular reaction and pathways.

One of the unique features of our knowledge-base design, i.e. DIO, is the adoption of the triplet view: intension,

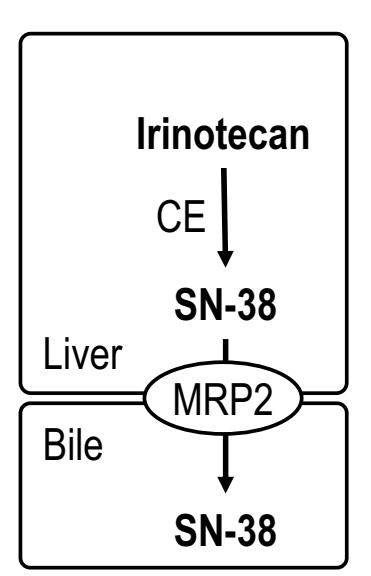

a) Pathway map representation

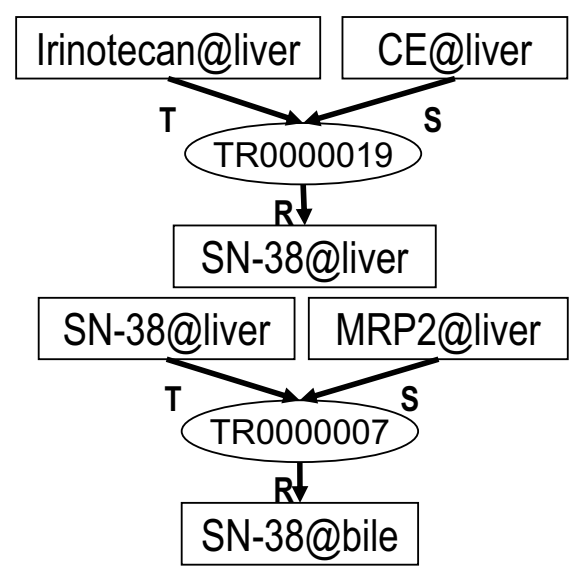

b) Modules of the pathway (Molecular events)

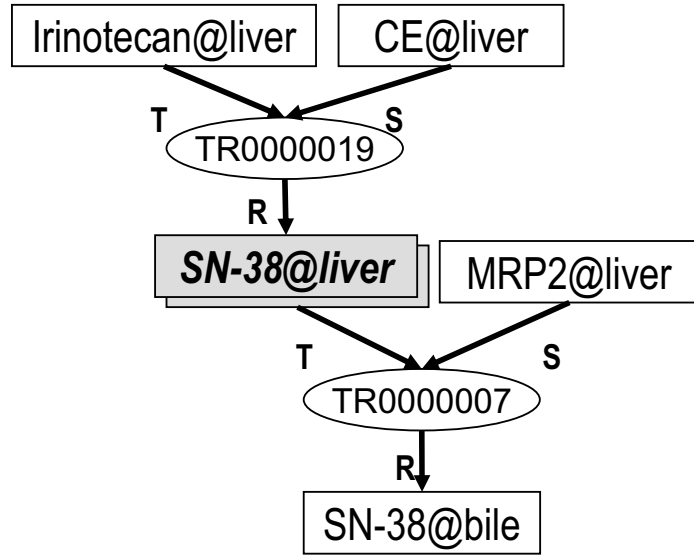

c) Connection of modules (Pathway)

Figure I

Decomposition of a pathway with primitive molecular events. a) Ordinary pathway map representation. b) Primitive molecular events including trigger (T), situator (S) and resultant (R). c) Aggregation of molecular events at SN-38@liver. Rectangles and ellipses are instances of continuants and processes, respectively, in $\mathrm{b}$ ) and $\mathrm{c}$ ). 
extension, attribute for molecular events and pathways as seen in Figure 2. Intension defines the kinds of molecular events and pathways as controlled vocabulary of processes. Attribute defines the components of molecular events and pathways as controlled vocabulary of continuants (see Figure 3 for a part of class hierarchy of controlled vocabulary for drug interaction). These controlled vocabularies are constructed from the viewpoints of "process" and "continuant" as proposed in Basic Formal Ontology (BFO) [9,10]. Molecular event objects are asserted in extension using the controlled vocabularies defined in intention and attributes. As for extension, prototype object modeling is adopted to represent pathway as aggregation of molecular events. This is because an infinite number of terms or classes are required to express all combinations of molecular events. We avoid this problem by treating pathways as dynamic objects deduced from prototype molecular event objects rather than treating them as instances of pathway classes.
Dynamic pathway reconstruction and drug interaction detection

The drug metabolic pathway, due to its dynamic nature, is difficult to define a priori in the manner seen in biomolecular metabolic pathways in the Kyoto Encyclopedia of Genes and Genomics (KEGG) [11]. Therefore, the OHA framework provides the Pathway Object Constructor (POC) and Drug Interaction Detector (DID). Pathway Object Constructor dynamically deduces pathways from the DIO, depending on contexts or situations. Drug Interaction Detector detects drug interaction candidates finding intersections from those generated pathways.

These inferences are mapped onto the Drug Interaction Ontology as hypothetical assertions. The generated pathways and detected drug interactions are asserted as aggregation of molecular events. The detected drug interactions can be segmentalized such as competitive inhibition, noncompetitive inhibition, and uncompetitive inhibition when binding site information is available. This is effec-



Figure 2

Overview of the Ontology-Driven Hypothetic Assertion (OHA) framework. Drug Interaction Ontology (DIO) provides triple views for events and pathways, namely, intension, extension, and attribute. White triangles in extension are molecular events such as CYP3A4 mediated metabolism of irinotecan. White rectangles and circles in attribute are molecules and organs respectively; the organs indicate the location of molecules. The gray triangles and arrowed dashed lines represent the hypothetic assertions generated by the OHA framework. The Pathway Object Constructor (POC) generates pathways by connecting molecular events. The Drug Interaction Detector (DID) detects drug interactions from the generated pathways and asserts the interactions as hypothetic assertions into DIO. 


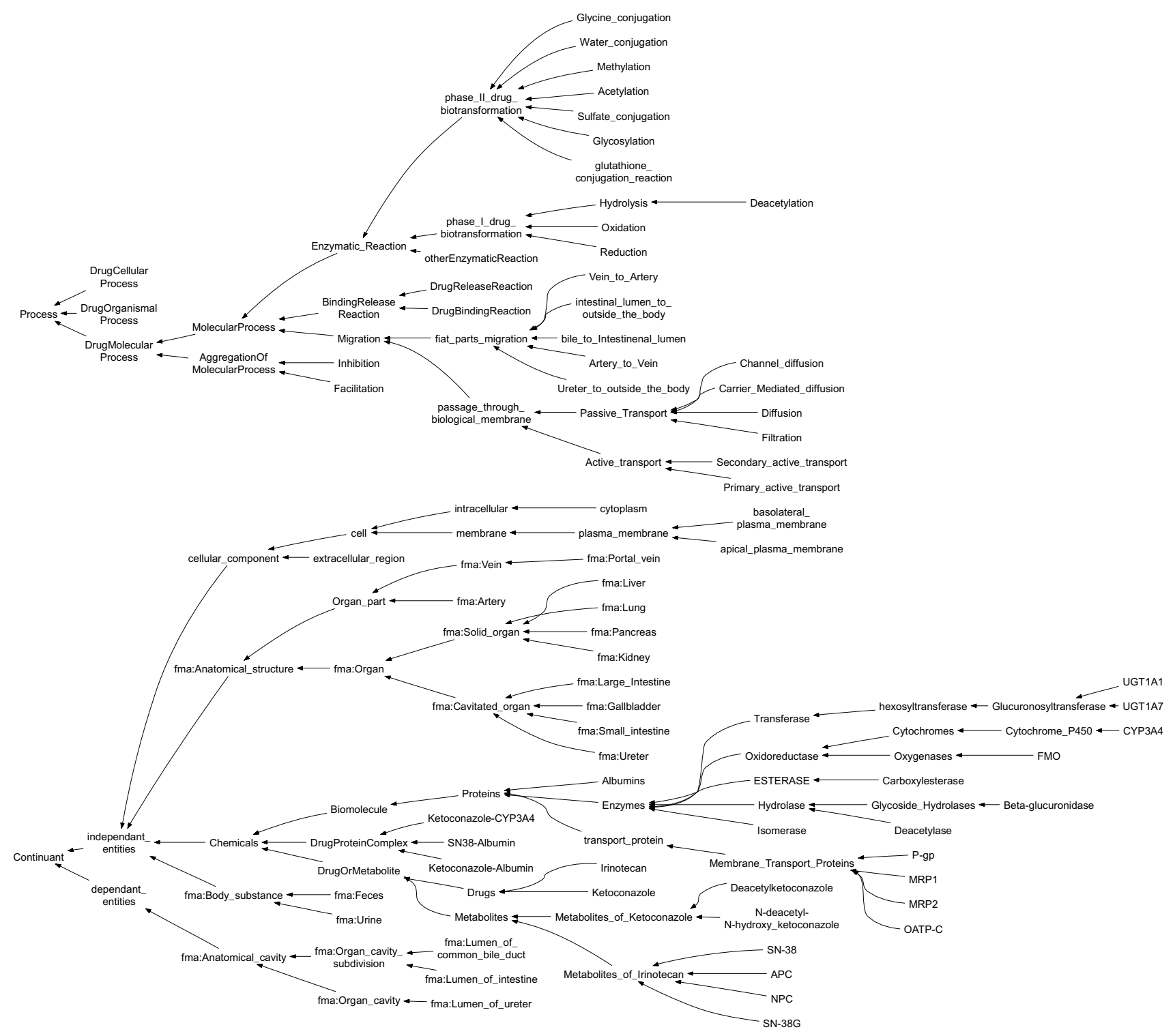

Figure 3

Class hierarchy for controlled vocabularies of DIO. Classes prefixed with "fma:" were references for the classes defined in the FMA. The class hierarchy was stored in "dio_event.owl". According to BFO, the controlled vocabulary was divided into two parts; process (e.g. events) and continuants (e.g. things). (This figure was generated using ontoviz plug-in of Protege with manual changes.).

tive to select an appropriate differential equation depending on the inhibition model.

\section{Numerical simulation}

In order to incorporate a quantitative simulation system into the OHA framework, the following two aspects were considered: a methodology for automatic conversion from a generated pathway to a quantitative simulation model, and a methodology for the validation of numerical simulation models by analysing the dependences of kinetic parameters and physiological parameters. By solving these two issues, the OHA framework can can be applied to in silico prediction of individual drug interactions for multiple-drug regimens, assuming that kinetic parameters and the initial enzyme concentration are roughly estimated by individual genetic variations and health indices of bio-markers.

A simulation model is automatically generated from a pathway generated by the Pathway Object Constructor 
and Drug Interaction Detector. The pathway is converted to intermediate model by merging organs and molecular events, respectively, to fit a given simulation model framework such as compartment model. Differential equations for the simulation model are generated from the intermediate model by converting the merged events to mathematical expression.

We used the parameter-parameter dependency analysis system (PPD Viewer) designed by Konagaya et al. [12], with high-throughput numerical simulation engine and interactive visualization tools developed on OBIGrid $[3,13-16]$. The system can predict the concentration/time profiles and those moment parameters such as area under the curve (AUC), area under the moment curve (AUMC), and mean resident time (MRT) when changing some kinetic parameters in the range of one thousandth to thousands of physiological conditions. The system visualizes dependencies among kinetic parameters and molecular concentrations in terms of moment parameters of a compound in target organs.

The generated simulation models and pharmacokinetic moment parameter values are mapped onto the Drug Interaction Ontology as hypothetical assertions. The simulation models are asserted as aggregations of objects representing terms and parameters in differential equations. Those objects have references to the components of the pathway objects from which the simulation models were generated. The moment parameter values are asserted with the drug interaction objects and the corresponding simulation model for the further analysis.

\section{Interaction between Irinotecan and Ketoconazole}

Irinotecan (CPT-11) is an anti cancer drug which is commonly used for colon and breast cancers [17]. Irinotecan is a prodrug of SN-38, anti neoplastic topoisomerase I inhibitor, and is bioactivatied by carboxyl esterase (CE) [17]. About $60 \%$ of irinotecan is excreated as unchanged drug from bile and kidney [18]. Irinotecan is also metabolised by CYP3A4 to form APC and NPC [17]. NPC is further metabolized by CE to form SN-38. SN-38 undergoes glucronidation by UGT1A1 to form the inactive glucronide, SN-38G [17]. In addition, it is known that the mutations on UGT1A1, UGT1A1*28 which decreases the expression of UGT1A1 enzyme down to $30 \%$, has strong relationship with some side effects of irinotecan $[19,20]$. Ketoconazole (KCZ) is an anti fungal drug and a well known inhibitor of CYP3A4. Ketoconazole undergoes extensive metabolism in the liver to form several metabolites [21,22]. About 2 to $4 \%$ of urinary radioactivity represents unchanged drug [22].
It has been reported that the inhibition of CYP3A4 by ketoconazole influences the metabolism of irinotecan, which results in $6 \% \mathrm{SN}-38$ increase [23].

\section{Results and discussion \\ Results}

Pathway generation and drug interaction detection

The pathways of intravenously administered irinotecan and orally administered ketoconazole were inferred as aggregation of molecular events by the Pathway Object Constructor. Figure 4 shows the generated irinotecan metabolic pathway object where irinotecan and its derivatives circulate through the veins, liver, bile, intestines, and portal vein, namely, the enterohepatic circulation, and are excreted through the kidneys or through the bile. These generated pathways are consistent with in vivo studies [17]. The generated pathway object is sound in the sense that the object is deduced from the Drug Interaction Ontology (DIO) represented by Web Ontology Language (OWL-DL) [24].

Interactions between intravenously administered irinotecan and orally administered ketoconazole were detected and asserted by the Drug Interaction Detector. The detected drug interactions and the hypothetic assertion are shown in Figure 5. The assertion contains four drug interactions; two of them concern "drug binding reaction" to albumin in veins (ddi2) and arteries (ddi3), and the rest of them concern "oxidation" by CYP3A4 (ddi0 and ddi1). The detected drug interaction concerning CYP3A4 (ddi0 and ddi1) has been confirmed by the literature on in vivo studies [23].

\section{Numerical simulation}

We evaluated the effects of drug interactions concerning CYP3A4 quantitatively with numerical simulations. Three simulations were performed: sole administration of irinotecan for a patient having UGT1A $1 * 1 /{ }^{*} 1$ (wild type), co-administration of irinotecan and ketoconazole for a patient having UGT1A1* $1 /{ }^{*} 1$, and sole administration of irinotecan for a patient with UGT1A $1 * 28 / * 28$ which decreases the expression of UGT1A1 down to $30 \%$ than UGT1A $1 * 1 /{ }^{*} 1$. Intravenous drip infusion $\left(125 \mathrm{mg} / \mathrm{m}^{2}, 90\right.$ min) was assumed for irinotecan, and oral administration (200 mg) was assumed for ketoconazole. Figure 6 shows the simulated concentration/time profiles of irinotecan, SN-38, APC, NPC, SN-38G in blood for the simulation of sole administration of irinotecan for a patient having UGT1A $1 * 1 / * 1$. The concentration/time profiles agree with experimental data reported by Slatter et al. [18].

Table 1 shows the comparisons of AUC and Cmax for the simulations. By the ketoconazole administration, the AUC of APC and NPC were decreased to $48.1 \%$ and $35.3 \%$, respectively. The AUC of SN-38 were increased 


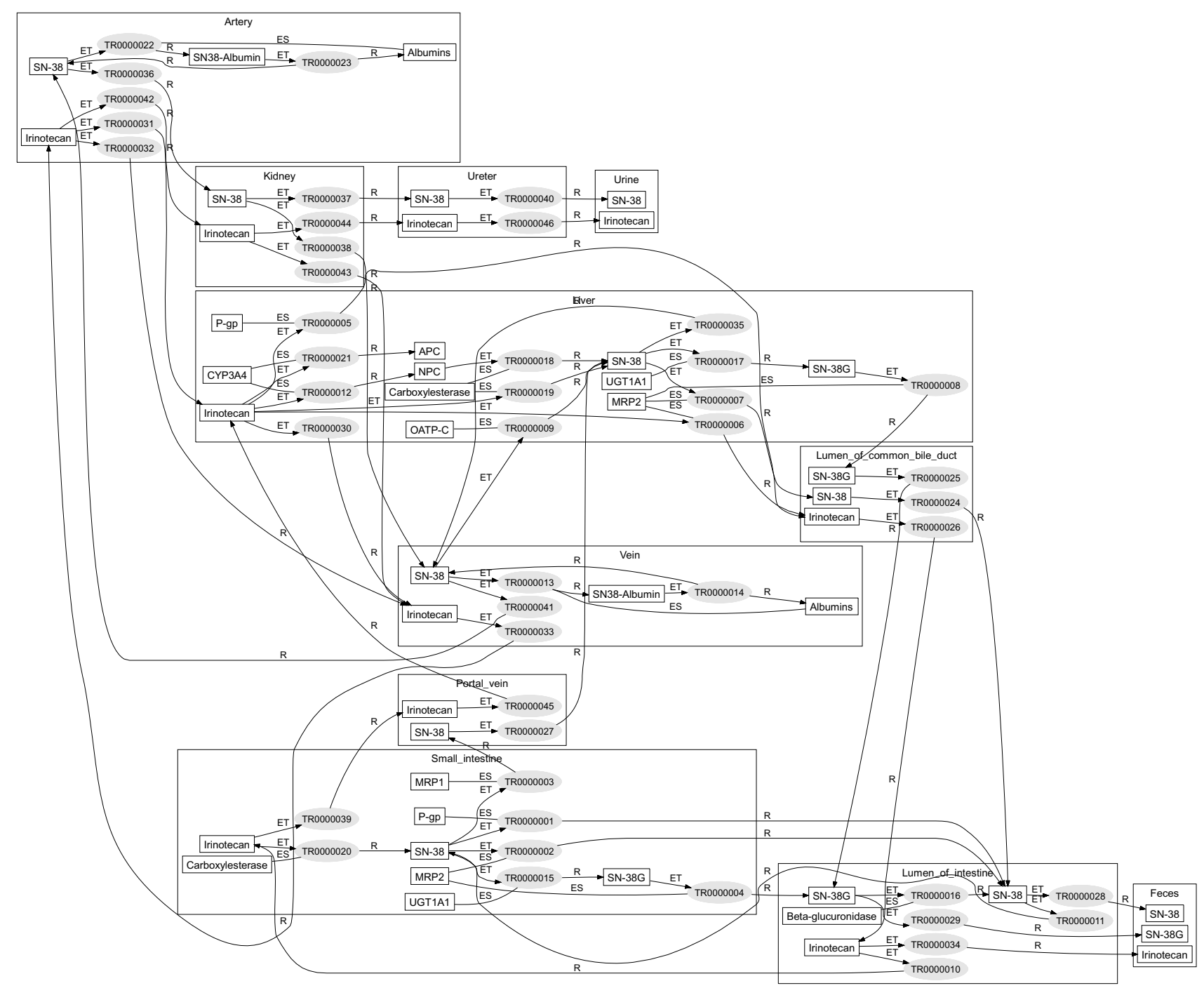

\section{Figure 4}

Inferred pathway of irinotecan. Small rectangles and ellipses are instances of continuants and molecular events, respectively. Large rectangles such as small intestine are the compartments. Molecular event and trigger, situator, and resultant were connected by solid lines. Each molecular event was connected by the shared continuants. For example, TR0000020 and TR0000002 in the small intestine were connected because they share the same instance of SN-38. This connection can be interpreted as that irinotecan is metabolized into SN-38 (TR0000020) and then SN-38 is transported to the intestinal lumen. (This figure was generated by our inference system.).

only to $108 \%$ by the ketoconazole administration. Similarly, the Cmax of APC and NPC were decreased to $25.6 \%$ and $20.2 \%$, respectively, whereas the Cmax of SN-38 was increased to $105 \%$ by the ketoconazole administration. On the other hand, in case of the UGT1A $1 * 28 /{ }^{*} 28$ mutation, the AUC and Cmax of $\mathrm{SN}-38$ were significantly increased: the AUC was increased to $208 \%$ and the Cmax was increased to $165 \%$. This implies that patients with UGT1A $1 * 28 / * 28$ may suffer severe side effects when the doses are same as patients with UGT1A $1 * 1 /{ }^{*} 1$. These results agree with the previously published experimental papers by Kehrer et al. [23], Sai et al. [19], and Ando et al. [20]. In addition, the simulation results were asserted into the ontology along with the simulation model.

\section{Discussion}

Pathway reconstruction and drug interaction detection

The OHA framework automatically generated pathways for co-administration of irinotecan and ketoconazole, and detected drug interactions. The generated pathways and two of the drug interactions involving CYP3A4 were confirmed by published papers. The detected interactions 


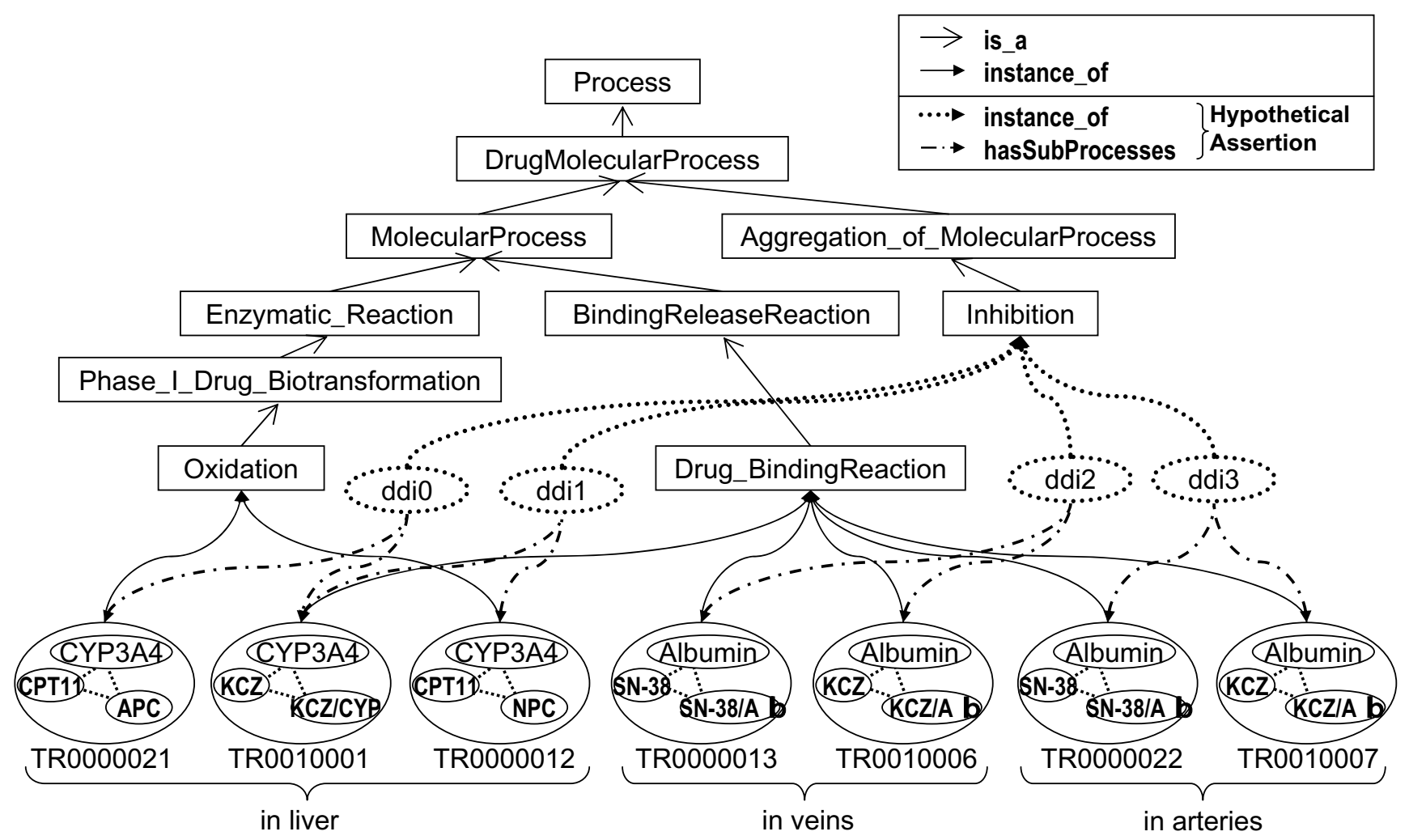

\section{Figure 5}

Dynamically asserted instances of interactions. Rectangles are owl classes corresponding to intension. Circles are owl instances corresponding to molecular events and attribute. Detected drug interactions were hypothetically asserted as owl instances (dotted circles) along with hasSubprocess properties (dotted and dashed edges).

with plasma albumin (ddi2 and ddi3) have not been reported in any literature as far as we know. One possible reason is that the effects of drug interactions may be negligible because of the high plasma albumin concentration compared to drug concentration. To distinguish serious drug interactions from those negligible ones, a qualitative evaluation, such as numerical simulation, is necessary.

\section{Numerical simulation}

The simulation model succeeded to reproduce Slatter's experimental data of irinotecan dosage to a large extent except for the Cmax of irinotecan blood concentration, cyclic fluctuation of irinotecan concentration and a bile cancer patient data.

The Cmax of drug concentrations in the simulation model strongly depend on renal clearance, bile clearance, and kinetic parameters of enzymes. Because these parameters were estimated from the recovery amount data in urine and feces by Slatter et al. [18] and in vitro experiments data reported as published papers [25-29], the excess of irinotecan's Cmax in the simulation model might be explained by the false peak in the experimental data which results from time lag of sampling

Another possibility is the effect of reabsorption in intestine. The time course of the experimental data indicate slight increase of SN-38G blood concentration in the period of 12 hours. This may result from the reabsorption of SN-38 thorough small intestine. In order to include the reabsorption process into the simulation, the kinetic parameters in bacterial flora in the intestine and amount/ time profile data of bile excretion are required.

Lastlym Slatter et al. reported that one patient with a bile cancer showed different bile and urine recovery pattern from other patients [18]. Virtual population [30] may be helpful to reconstruct this behaviour.

\section{Conclusion}

Deduction of hypothetic drug interactions from the Drug Interaction Ontology was demonstrated for an irinotecan plus ketoconazole regimen. The prototype system detected four drug interactions. Two of them concerned 


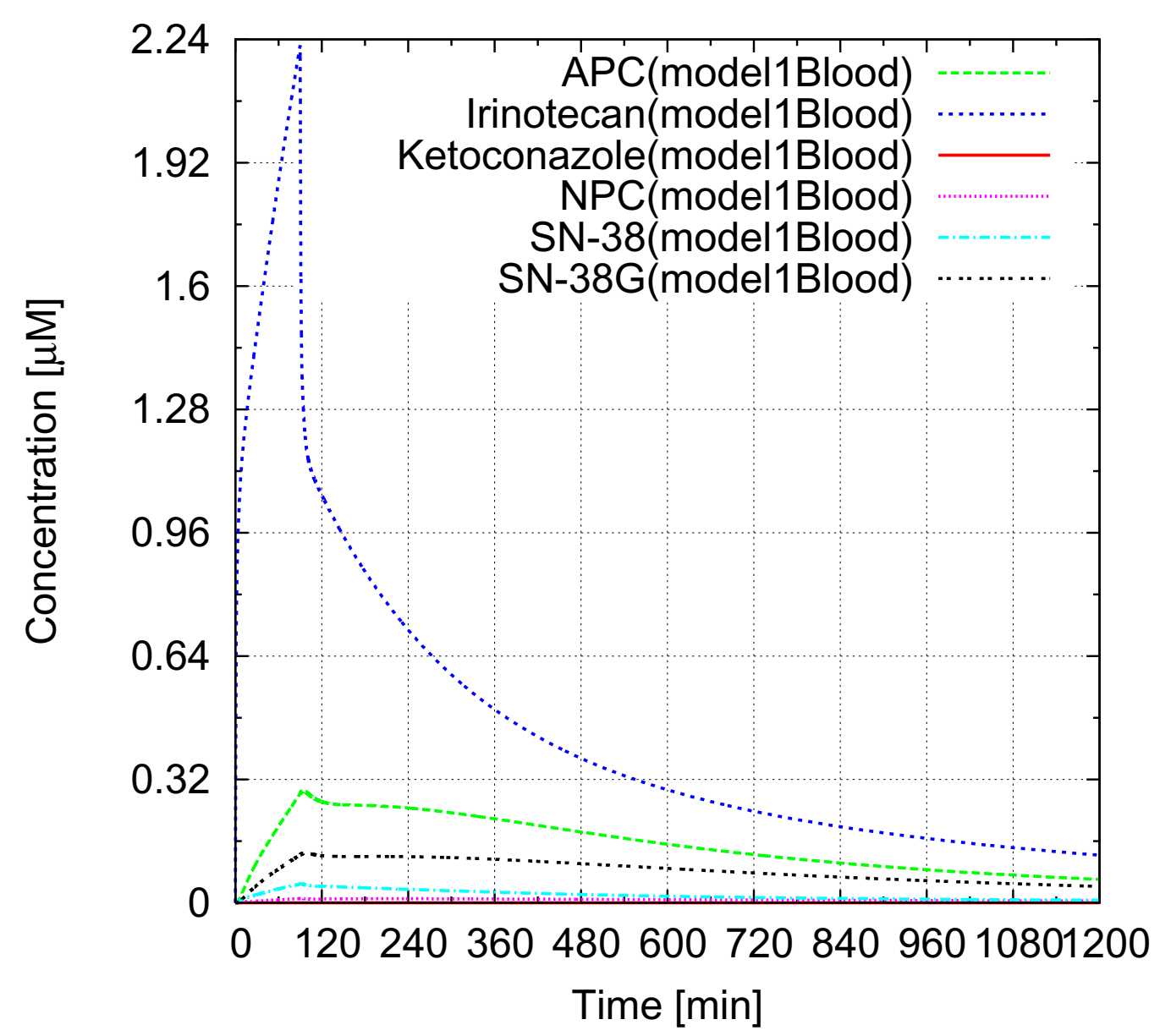

\section{Figure 6}

Blood Concentration/time profile of irinotecan, SN-38, APC, NPC, and SN-38 in blood compartment for solo administration of irinotecan. The drug concentrations in blood without ketoconazole administration was estimated by numerical simulation. Intravenous drip infusion ( $125 \mathrm{mg} / \mathrm{m}^{2}, 90 \mathrm{~min}$ ) was assumed for irinotecan administration.

cytochrome p450 (CYP3A4) and were consistent with known drug interactions. The other two concerned albumin, the effects of the interactions would be negligible as long as the drug concentration is low. We then quantitatively examined the effect of the drug interactions concerned CYP3A4 and the effect of genetic variation UGT1A $1 * 28$ using numerical simulation. The numerical simulation indicates that the drug interaction has only a limited effect on the pharmacokinetics of SN-38 for an irinotecan plus ketoconazole regimen. However, the genetic variation on UGT1A1 showed two-fold increase of SN38's AUC.

Finally, in order to realize in silico prediction of drug interactions, the following future works are remained: expansion of the Drug Interaction Ontology for other drugs, incorporation of virtual population model for generic var- iation analysis, and refinements of the pathway generation rules, drug interaction detection rules, and the numerical simulation models.

\section{Methods \\ The OHA framework}

We implemented the following prototype system to prove the concept of the OHA framework. The system was designed to predict potential drug interactions occurring after concomitant administration of irinotecan and ketoconazole by comparing the drug metabolic pathways generated from primitive molecular events. Figure 7 shows an overview of the prototype system. The system consists of the knowledge base for DIO, the inference programs of the Pathway Object Constructor and Drug Interaction Detector, simulation model generator, and simulation engine. 
Table I: AUC and Cmax of blood concentration/time profile obtained by numerical simulations.

\begin{tabular}{|c|c|c|c|c|c|}
\hline & Irinotecan & APC & NPC & SN-38 & SN-38G \\
\hline$A \cup C_{K C Z-}[\mathrm{nmol} \cdot \mathrm{min} / \mathrm{ml}]$ & 672 & 233 & 14.2 & 29.8 & 137 \\
\hline$C \max _{K C Z-}[\mu \mathrm{M}]$ & 2.22 & 0.290 & 0.0113 & 0.0491 & 0.129 \\
\hline$A \cup C_{K C Z+}[\mathrm{nmol} \cdot \mathrm{min} / \mathrm{ml}]$ & 721 & 112 & 5.01 & 32.2 & 147 \\
\hline $\mathrm{Cmax}_{K C Z+}[\mu \mathrm{M}]$ & 2.29 & 0.0741 & 0.00228 & 0.0515 & 0.136 \\
\hline$A \cup C_{K C Z+} / A \cup C_{K C Z-}[\%]$ & 107 & 48.1 & 35.3 & 108 & 107 \\
\hline $\mathrm{Cmax}_{\mathrm{KCZ}} / \mathrm{Cmax} \operatorname{mCZ}[\%]$ & 103 & 25.6 & 20.2 & 105 & 105 \\
\hline$A U C_{U G T}[\mathrm{nmol} \cdot \mathrm{min} / \mathrm{ml}]$ & 672 & 233 & 14.2 & 62.1 & 85.5 \\
\hline $\mathrm{Cmax}_{U G T}[\mu \mathrm{M}]$ & 2.22 & 0.290 & 0.0113 & 0.0812 & 0.0693 \\
\hline$A \cup C_{U G T} / A \cup C_{K C Z-[}[\%]$ & 100 & 100 & 100 & 208 & 62.4 \\
\hline $\mathrm{Cmax}_{U G T} / \mathrm{Cmax}_{\mathrm{KCZ}-}[\%]$ & 100 & 100 & 100 & 165 & 53.7 \\
\hline
\end{tabular}

AUC and $C$ max values for solo administration of irinotecan $\left(A \cup C_{K C Z-}, \operatorname{Cmax}_{K C Z}\right)$, co-administration of irinotecan and ketoconazole ( $A U C_{K C Z+}$, $\left.\mathrm{Cmax}_{\mathrm{KCZ}}\right)$, and sole administration of irinotecan with patients having UGTIAI*28/*28 (AUC $\left.\mathrm{UGT}, \mathrm{Cmax}_{\mathrm{UGT}}\right)$ were obtained.

DIO, shown in Figure 2, was written in OWL-DL; the controlled vocabularies of process and continuant were implemented as OWL class hierarchy, and extension and part of attributes, including molecules and organs, were implemented as OWL instances. The molecular event objects in extension were represented by OWL instances and OWL properties. The ontology referred to other taxonomies and ontologies for well-established vocabularies of biochemical terms, anatomical entities and properties. This enabled the reduction of our ontology construction cost and to concentrate our efforts on the information specific to drug interaction.

The ontology for irinotecan and ketoconazole was built with Protege [31]. The information about these drugs has been collected from various articles [17,22,32-38]. The total number of classes and instances of the current DIO is 178 and 143, respectively. Approximately two thirds of the classes were mapped to the Unified Medical Language System (UMLS) [39] and/or Foundational Model of Anatomy (FMA) $[40,41] ; 106$ classes were mapped to the UMLS; 32 classes were mapped to the FMA. The class hierarchy is shown in Figure 3. Twenty-six properties were imported from the Open Biomedical Ontology Relation Ontology (OBO Relation) [42]; and five properties were newly defined to implement molecular event objects as OWL instances (Table 2). The ontology OWL files: dio_event.owl, dio_cpt-11, and dio_KCZ.owl, are available as additional files $1,2,3$.

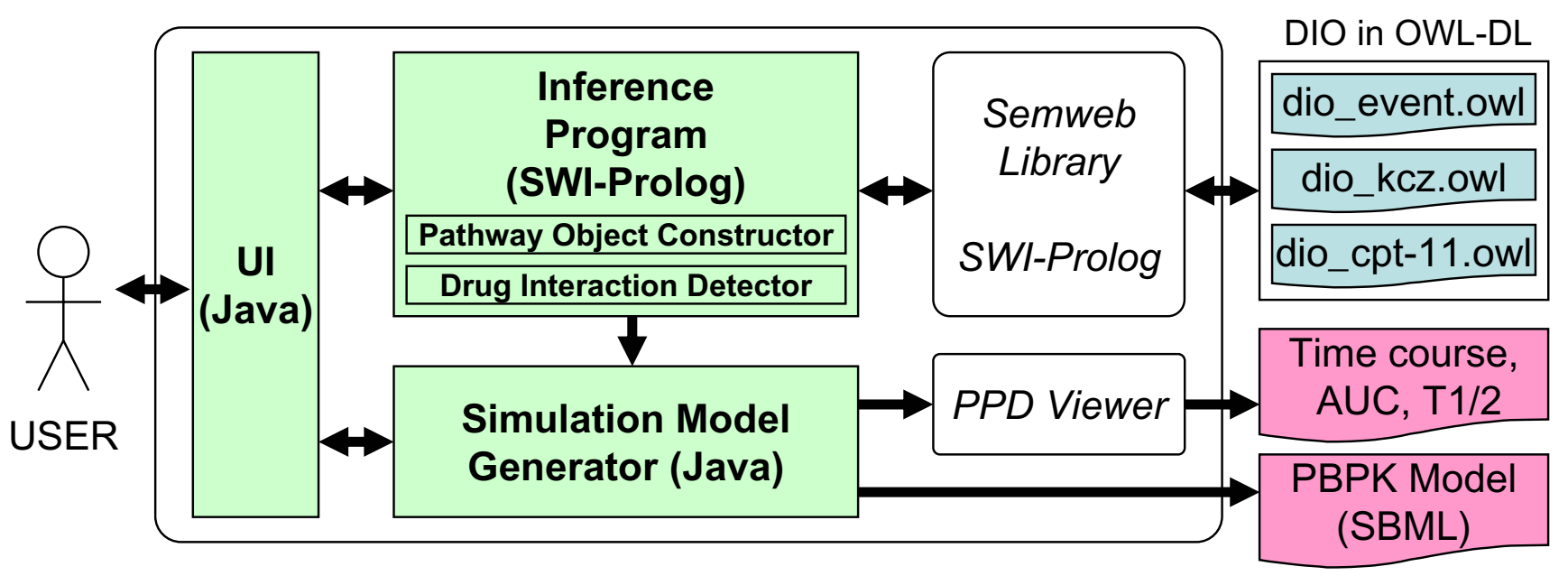

\section{Figure 7}

Overview of the prototype system. User interface (UI), inference program, and visualization program were implemented in Prolog and JAVA. The ontology (DIO) was divided into three OWL files. I) Class hierarchy and commonly used instances are stored in dio_event.owl. 2) Instances about irinotecan and its pathway are stored in dio_cpt-I I.owl. 3) Instances about ketoconazole and its pathway are stored in dio_KCZ.owl. 
Table 2: The major properties of molecular event objects in OWL-DL.

\begin{tabular}{lcc}
\hline Properties & Domain (class) & Range (class) \\
\hline $\begin{array}{l}\text { oboRel:relationship } \\
\text {-oboRel:located in }\end{array}$ & $\begin{array}{c}\text { Process, Continuant } \\
\text { independent_entities }\end{array}$ & Process, Continuant \\
-oboRel:has_participant & Process & Independent_entities, fma:Anatomical_cavity \\
-- hasResultantParticipant & Process & Independent_entities \\
- - hasTriggerParticipant & Process & Independent_entities \\
-- hasSituatedParticipant & Process & Independent_entities \\
\hline hasSubProcesses & & Independent_entities \\
occurred_in & Process & Process \\
\end{tabular}

The relations among molecular events, attributes (continuants) and drug interactions shown in Figure 2 were implemented as $O W L$ properties. We defined five properties for implementing molecular event objects in OWL-DL in addition to the properties of OBO Relation ontology. Three properties for resultant, trigger, and situated participants were defined as sub properties of oboRel:has_participant, inheriting the definition of the super property. Note that classes for Domain and Range of properties are denoted by the controlled vocabulary defined in Figure 3.

The inference programs of the Pathway Object Constructor and Drug Interaction Detector were implemented with SWI-Prolog [43] and the Semweb library. The Semweb library enables the system to access OWL and RDF files as Prolog clauses. All logical inferences, including pathway generation and drug interaction detection, were implemented in Prolog.

Systems for simulation model generation, and pathway visualization were implemented in JAVA. These systems access DIO through SWI-Prolog semweb library to get detailed information of the generated pathways. The simulation models were generated in the form of SBML, then translated in the form of Octave. Graphviz [44], a graph visualization program designed by Gansner et al., was used to visualize pathways generated by the inference programs. The PPD Viewer designed by Azuma et al. [12] was used for numerical simulation.

\section{Numerical simulation model for co-administration of Irinotecan and Ketoconazole}

In order to increase predictive performance, a simplified pathway was used for the generation of simulation models from the viewpoint of the trade off between model complexity and data availability. Reabsorption through small intestine and reactions concerning albumin were omitted due to the lack of information. Furthermore, enzymatic reactions involved metabolism of ketoconazole were integrated into a single enzymatic reaction based on the work of Chien et al. [29].

A simulation model was automatically generated from the simplified pathway. The organs and tissues were integrated into 8 compartments, i.e. blood (including rapidly equilibrating tissues: artery, heart, kidneys, lung, and veins), liver, GI (gastrointestinal consists of large intestine, small intestine, portal vein, and stomach), adipose, NET (non-eliminating tissue such as skin and muscle), GI lumen, bile lumen, and urine. Michaelis-Menten equations were used for all enzymatic reactions. We used a competitive Michaelis-Menten inhibition model for this simulation as used for midazolam and ketoconazole inhibition by Chien et al. [29]. For drugs and their metabolites in blood, adipose, NET, GI, and liver, the following equations apply:

$$
\begin{aligned}
\frac{d C_{1 D}}{d t}= & \left\{-C L_{u, r, D} f_{B D} C_{1 D}+\left(Q_{2}+Q_{3}\right) \frac{C_{2 D}}{K p_{2} D}+Q_{5} \frac{C_{5 D}}{K p_{5 D}}\right. \\
+ & \left.Q_{4} \frac{C_{4 D}}{K p_{4 D}}-\left(Q_{2}+Q_{3}+Q_{4}+Q_{5}\right) C_{1 D}\right\} / V_{1} \\
& \frac{d C_{4 D}}{d t}=\left(Q_{4} C_{1 D}-Q_{4} \frac{C_{4 D}}{K p_{4} D}\right) / V_{4} \\
& \frac{d C_{5 D}}{d t}=\left(Q_{5} C_{1 D}-Q_{5} \frac{C_{5 D}}{K p_{5 D}}\right) / V_{5} \\
& \frac{d C_{3 I M}}{d t}=\left(Q_{3} C_{1 I M}-Q_{3} \frac{C_{3 I M}}{K p_{3 I M}}\right) / V_{3} \\
\frac{d C_{3 K C Z}}{d t}= & \left(k a_{K C Z} X_{K C Z, G I L u m e n}+Q_{3} C_{1 K C Z}-Q_{3} \frac{C_{3 K C Z}}{K p_{3 K C Z}}\right) / V_{3}
\end{aligned}
$$




$$
\begin{aligned}
\frac{d C_{2 I}}{d t}= & \left\{Q_{2} C_{1 I}+Q_{3} \frac{C_{3 I}}{k p_{3 I}}-\left(Q_{2}+Q_{3}\right) \frac{C_{2 I}}{k p_{2 I}}\right. \\
& -\left(C L_{u, b, I}+\frac{V m a x_{a} a l p h a_{a} V_{2}}{k m_{a}+\frac{f b_{I} C_{2 I}}{K p_{2 I}}}+\frac{\operatorname{Vmax}_{c} a l p h a_{c} V_{2}}{k m_{C}\left(1+\frac{f b_{K C Z} C_{2 K C Z}}{K p_{2} K C Z K i_{A P C}}\right)+\frac{f b_{I} C_{2 I}}{K p_{2 I}}}\right. \\
& \left.\left.+\frac{\operatorname{Vmax}_{d} a l p h a_{d} V_{2}}{k m_{d}\left(1+\frac{f b_{K C Z} C_{2 K C Z}}{K p_{2 K C Z} K i_{N P C}}\right)+\frac{f b_{I} C_{2 I}}{K p_{2 I}}}\right) \frac{f b_{I} C_{2 I}}{K p_{2 I}}\right\} / V_{2}
\end{aligned}
$$

$$
\begin{aligned}
& \frac{d C_{2 A P C}}{d t}=\left\{Q_{2} C_{1 A P C}+Q_{3} \frac{C_{3 A P C}}{K p_{3 A P C}}+\frac{V m_{c} a_{C} a l p h a_{c} V_{2}}{K m_{C}\left(1+\frac{f b_{K C Z} C_{2 K C Z}}{K p_{2} K C Z K i_{A P C}}\right)+\frac{f b_{I} C_{2 I}}{K p_{2 I}}} \frac{f b_{I} C_{2 I}}{K p_{2 I}}\right. \\
& \left.-\left(Q_{2}+Q_{3}\right) \frac{C_{2 A P C}}{K p_{2} A P C}-C L_{u, b, A P C} \frac{f b_{A P C} C_{2 A P C}}{K p_{2 A P C}}\right\} / V_{2}
\end{aligned}
$$

$$
\begin{aligned}
& \frac{d C_{2 N P C}}{d t}=\left\{Q_{2} C_{1 N P C}+Q_{3} \frac{C_{3 N P C}}{K p_{3 N P C}}+\frac{V m_{a x} a l p h a_{d} V_{2}}{K m_{d}\left(1+\frac{f b_{K C Z} C_{2 K C Z}}{K p_{2} K C Z^{K i_{N P C}}}\right)+\frac{f b_{I} C_{2 I}}{K p_{2 I}}} \frac{f b_{I} C_{2 I}}{K p_{2 I}}\right. \\
& \left.-\left(Q_{2}+Q_{3}\right) \frac{C_{2 N P C}}{K p_{2 N A P C}}-\left(C L_{u, b, N P C} \frac{V \max _{b} a l p h a_{b} V_{2}}{\left.K m_{b}+\frac{f b_{N P C} C_{2 N P C}}{K p_{2 N P C}}\right)}\right) \frac{f b_{N P C} C_{2 N P C}}{K p_{2 N P C}}\right\} / V_{2}
\end{aligned}
$$

$$
\begin{aligned}
& \frac{d C_{2 S N-38}}{d t}=\left\{Q_{2} C_{1 S N-38}+Q_{3} \frac{C_{3 S N-38}}{K p_{3 S N-38}}-\left(Q_{2}+Q_{3}\right) \frac{C_{2 S N-38}}{K p_{2 S N-38}}\right. \\
& +\frac{V m_{a x} a l p h a_{a} V_{2}}{K m_{a}+\frac{f b_{I} C_{2 I}}{K p_{2 I}}} \frac{f b_{I} C_{2 I}}{K p_{2 I}}+\frac{V m^{2} x_{b} a_{p h} a_{b} V_{2}}{K m_{b}+\frac{f b_{N P C} C_{2 N P C}}{K p_{2 N P C}}} \frac{f b_{N P C} C_{2 N P C}}{K p_{2 N P C}} \\
& \left.-\left(C L_{u, b, S N-38}+\frac{V_{m a x} \text { alpha }_{f} V_{2}}{K m_{f}+\frac{f b_{S N-38} C_{2 S N-38}}{K p_{2 S N-38}}}\right) \frac{f b_{S N-38} C_{2 S N-38}}{K p_{2 S N-38}}\right\} / V_{2}
\end{aligned}
$$

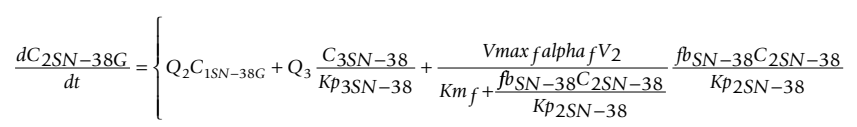

$$
\begin{aligned}
& \left.-\left(Q_{2}+Q_{3}\right) \frac{C_{2 S N-38 G}}{K p_{2} S N-38 G}-C L_{u, b, S N-38 G} \frac{f b_{S N}-38 G C_{2} S N-38 G}{K p_{2 S N}-38 G}\right\} / V_{2}
\end{aligned}
$$

$$
\begin{gathered}
\frac{d C_{2 K C Z}}{d t}=\left\{Q_{2} C_{1 K C Z}+Q_{3} \frac{C_{3 K C Z}}{K p_{3 K C Z}}-\left(Q_{2}+Q_{3}\right) \frac{C_{2 K C Z}}{K p_{2 K C Z}}\right. \\
\left.-\frac{\operatorname{Vmax}_{e} a l p h a_{e} V_{2}}{K m_{e}+\frac{f b_{K C Z} C_{2 K C Z}}{K p_{2 K C Z}}} \frac{f b_{K C Z} C_{2 K C Z}}{K p_{2 K C Z}}\right\} / V_{2}
\end{gathered}
$$

Here $Q p_{n^{\prime}}$ and $V_{n}(n=1$ to 5$)$ are blood flows and volume of compartments, respectively. The subscripts $n$ stands for compartments: $n=1,2,3,4$ and 5 represents blood, liver, GI, adipose, and NET, respectively. $K p_{n s^{\prime}}$ and $C_{n s}(n=1$ to $5, s=\mathrm{D}, \mathrm{I}, \mathrm{KCZ}, \mathrm{IM}, \mathrm{APC}, \mathrm{NPC}, \mathrm{SN}-38$, and SN-38G) are tissue-to-blood concentration ratios, and blood concentrations, respectively. The subscripts $s$ stands for drugs and their metabolites: $s=\mathrm{D}, \mathrm{I}, \mathrm{KCZ}$, IM, APC, NPC, SN-38, and $\mathrm{SN}-38 \mathrm{G}$ represents drugs and metabolites, irinotecan, ketoconazole, metabolites of irinotecan, APC, NPC, SN38, and SN-38, respectively. $C L_{u, r, s} C L_{u, b, s^{\prime}}$ and $f_{B s}(s=\mathrm{D}, \mathrm{I}$, KCZ, IM, APC, NPC, SN-38, and SN-38G) are renal clearance for unbound drugs, bile clearance for unbound drugs, and blood unbound fraction, respectively. $k a_{K C Z}$ and $X_{K C Z ; G I L u m e n}$ are absorption rate constant of ketoconazole and amount of ketoconazole in the GI luminal compartment, where ketoconazole is absorbed. $V \max _{m^{\prime}} \mathrm{km}_{m^{\prime}}$ and $\alpha_{m}(m=\mathrm{a}, \mathrm{b}, \mathrm{c}, \mathrm{d}, \mathrm{e}$, and $\mathrm{f})$ are Vmax, Michaelis constant, and expression amount of enzyme, respectively. The subscripts $m$ stands for enzymatic reactions: $\mathrm{m}=\mathrm{a}, \mathrm{b}, \mathrm{c}, \mathrm{d}$, $e$, and $f$ represents metabolism of irinotecan to form $\mathrm{SN}$ 38 by CE, metabolism of NPC to form SN-38 by CE, oxidation of irinotecan to form APC by CYP3A4, oxidation of irinotecan to form NPC by CYP3A4, glucronidation of SN-38 to form SN-38G by UGT1A1, and metabolism of ketoconazole by CYP3A4, respectively. $K i_{A P C}$ and $K i_{N P C}$ are inhibition constants. During the drip infusion of irinotecan, the following equation apply for blood concentration of irinotecan.

$$
\begin{aligned}
\frac{d C_{1 I}}{d t}= & \left\{k 0_{I}-C L_{u, r, I} f_{B I} C_{1 I}+\left(Q_{2}+Q_{3}\right) \frac{C_{2 I}}{K p_{2 I}}+Q_{5} \frac{C_{5 I}}{K p_{5 I}}\right. \\
& \left.+Q_{4} \frac{C_{4 I}}{K p_{4 I}}-\left(Q_{2}+Q_{3}+Q_{4}+Q_{5}\right) C_{1 I}\right\} / V_{1}
\end{aligned}
$$

Here $k 0_{I}$ is a constant for the velocity of drip infusion.

The values of physiologic parameters, kinetic parameters, and inhibition constants and administration parameters for the simulation model are shown in Table 3, 4, 5 and 6 respectively. The values of these parameters were based on published papers except enzyme expression parameters ( $\alpha$ ) and tissue-to-blood concentration ratios (Kp). The values for $\alpha$ and Kp were obtained by fitting the simulation result to the experimental data published by Slatter et al. [18].

\section{Competing interests}

The authors have received no financial support or other benefits from commercial sources for the work reported in the manuscript, and no other financial interests that any of the authors may have could create a potential conflict 
Table 3: Kinetic parameters for the protein binding, tissue distribution, and urinary and biliary excretion of ketoconazole, irinotecan, and their metabolites.

\begin{tabular}{|c|c|c|c|c|c|c|c|}
\hline Compound & $f_{B}$ & $K p_{G l}$ & $K p_{\text {Liver }}$ & $K p_{\text {Adipose }}$ & $K p_{N E T}$ & $C L_{U, R}[\mathrm{~mL} / \mathrm{min} / \mathrm{kg}]$ & $C L_{U, B}[\mathrm{~mL} / \mathrm{min} / \mathrm{kg}]$ \\
\hline Irinotecan & 0.37 & 1.00 & 1.00 & 10.0 & 3.00 & 6.15 & 10.6 \\
\hline APC & 0.37 & 1.00 & 1.00 & 1.50 & 0.06 & 1.47 & 5.45 \\
\hline NPC & 0.37 & 1.00 & 1.00 & 6.00 & 2.00 & 1.49 & 14.5 \\
\hline SN-38 & 0.05 & 1.00 & 1.00 & 2.00 & 0.70 & 9.91 & 103 \\
\hline SN-38G & 1.00 & 1.00 & 1.00 & 2.80 & 0.08 & 1.44 & 2.03 \\
\hline $\mathrm{KCZ}$ & 0.01 & 1.00 & 1.00 & 15.0 & 1.50 & 130 & - \\
\hline
\end{tabular}

Blood unbound fraction $\left(f_{B}\right)$ values for irinotecan and SN-38 were obtained from the published paper by Oliver et al. [45]. The $f_{B}$ values for NPC and APC were assumed to be the same as that of Irinotecan. $f_{B}$ value for SN-38G was assumed to be I.00. Urinary and biliary clearances $\left(C L_{U, R}\right.$, $\left.C L_{U, B}\right)$ were defined for unbound blood concentration. $C L_{U, R}$ and $C L_{U, B}$ values for irinotecan, APC, SN-38, SN-38G were calculated using data from the publish paper [18]. Kp values were determined so that the simulation concentration/time profile fits to the experimental data from Slatter et al. [18].

Table 4: Kinetic parameters for metabolic enzymes.

\begin{tabular}{llllccc}
\hline Enzyme & Substrate & Product & $K_{m}[\mu \mathrm{M}]$ & $V_{\max }[\mathrm{pmol} / \mathrm{min} / \mathrm{mg}$ protein $]$ & $\alpha[\mathrm{mg}$ protein/g tissue $]$ & $V_{\max } \cdot \alpha / K_{m}[\mathrm{~mL} / \mathrm{min} / \mathrm{g}$ tissue $]$ \\
\hline Carboxylesterase & Irinotecan & $\mathrm{SN}-38$ & 2.30 & 2.11 & 128 & 0.117 \\
Carboxylesterase & NPC & SN-38 & 2.30 & 2.11 & 128 & 0.117 \\
CYP3A4 & Irinotecan & APC & 18.4 & 26.0 & 73.3 & 0.104 \\
CYP3A4 & Irinotecan & NPC & 48.2 & 74.1 & 71.7 & 0.0180 \\
UGTIAI & SN-38 & SN-38G & 3.80 & 50.8 & 750 & 10.0 \\
\hline CYP3A4 & KCZ & MOK & 0.00810 & 12.5 & 44.3 & 68.4 \\
\hline
\end{tabular}

Because of the lack of experimental data, $K_{m}$ and $V_{\max }$ values for metabolism of NPC to SN-38 by CE were assumed to be the same as those for metabolism of irinotecan to SN-38 by CE. $K_{m}$ and $V_{\max }$ values for other reactions were obtained from the published papers [25-29]. Values of unknown parameter $\alpha$ were determined so that the simulation concentration/time profile fit to the experimental data published by Slatter et al. [18]. The relatively high fitted value for $\alpha$ in the glucronidation of SN-38 may result from the increase in UGTIAI expression as drug response. The relatively low value for $\alpha$ in the oxidation of irinotecan to NPC by CYP3A4 suggests a competition in this reaction with the oxidation of irinotecan to APC by CYP3A4.

Table 5: Physiologic parameters.

\begin{tabular}{lcc}
\hline Organ & Blood flow rate $[\mathrm{mL} / \mathrm{min} / \mathrm{kg}]$ & Volume $[\mathrm{mL} / \mathrm{kg}]$ \\
\hline Blood & 61.1 & 51.0 \\
Liver & 5.79 & 32.3 \\
GI & 13.4 & 32.1 \\
Adipose & 4.45 & 204 \\
NET & 37.4 & 681 \\
\hline
\end{tabular}

The physiologic parameters were obtained from the work by Willmann et al. [30]. These values assumes Caucasian male with body weight of $73 \mathrm{~kg}$ and height of $176 \mathrm{~cm}$.
Table 6: Parameters for drug interactions and drug administrations.

\begin{tabular}{lc}
\hline Parameters & Values \\
\hline$K i_{A P C}$ & $1.40 * 10^{-3}$ \\
$K i_{N P C}$ & $5.94 * 10^{-4}$ \\
$k a_{K C Z}\left[\mathrm{~min}^{-1}\right]$ & $1.83 * 10^{-2}$ \\
dose $_{\text {lrinotecan }}[\mathrm{nmol} / \mathrm{kg}]$ & $4.86 * 10^{3}$ \\
$t_{\text {dose,lrinotecan }}[\mathrm{min}]$ & 90 \\
dose $_{K C Z}[\mathrm{nmol} / \mathrm{kg}]$ & $5.16 * 10^{3}$ \\
\hline
\end{tabular}

$K i_{A P C}$ and $K i_{N P C}$ were calculated from the experimental data reported by Haaz et al. $[25,26] . k a_{K C Z}$ was obtained the from published paper [29]. 
of interest or the appearance of a conflict of interest with regard to the work.

\section{Authors' contributions}

Takeshi Arikuma carried out the machine inference studies, participated in Drug Interaction Ontology development, participated in numerical simulation studies and drafted the manuscript. Sumi Yoshikawa carried out the ontology studies, participated in machine inference studies, and helped to draft the manuscript. Ryuzo Azuma carried out the numerical simulation and visualization studies. Kazumi Matsumura extracted knowledge from the articles for the Drug Interaction Ontology when she worked for RIKEN GSC. Kentaro Watanabe developed the original drug-interaction prediction system when he studied in TITECH. Akihiko Konagaya conceived of the study, partcipated in its design and coordination, and helped to draft the manuscript.

\section{Additional material}

\section{Additional file 1}

owl file for dio_event ontology: This file includes all OWL classes and properties defined for the drug interaction ontology (DIO).

Click here for file

[http://www.biomedcentral.com/content/supplementary/1471-

2105-9-S6-S11-S1.owl]

\section{Additional file 2}

owl file for dio_cpt-11 ontology: This file includes all OWL instances of reactions defined for irinotecan.

Click here for file

[http://www.biomedcentral.com/content/supplementary/1471-

2105-9-S6-S11-S2.owl]

\section{Additional file 3}

owl file for dio_kcz ontology: This file includes all OWL instances of reactions defined for ketoconazole.

Click here for file

[http://www.biomedcentral.com/content/supplementary/14712105-9-S6-S11-S3.owl]

\section{Acknowledgements}

The authors give special thanks to Dr. Fumikazu Konishi, Mr. Shingo Ohki and Mr. Ryo Umetsu in RIKEN GSC for their contributions in providing the CONDOR pool and visualization tools on the Open Bioinformatics Grid (OBIGrid).

The computations in this work were performed using the Open Bioinformatics Grid (OBIGrid) [13].

This article has been published as part of BMC Bioinformatics Volume 9 Supplement 6, 2008: Symposium of Computations in Bioinformatics and Bioscience (SCBB07). The full contents of the supplement are available online at http://www.biomedcentral.com//47|-2105/9? issue=S6.

\section{References}

I. Okuda H, Ogura K, Kato A, Takubo H, Watanabe T: A possible mechanism of eighteen patient deaths caused by interactions of sorivudine, a new antiviral drug, with oral 5-fluorouracil prodrugs. J Pharmacol Exp Ther 1998, 287(2):791-799.

2. Baker CJ, Cheung KH: Semantic Web: Revolutionizing Knowledge Discovery in the Life Sciences Springer; 2007.

3. Konagaya $A$ : OBIGrid: towards the $B a$ for sharing resources, services and knowledge for bioinformatics. 4th International Workshop on Biomedical Computations on the Grid (BioGrid'06), Singapore 2006.

4. Tsukamoto $Y$, Kato $Y$, Ura M, Horii I, Ishitsuka H, Kusuhara H, Sugiyama $Y$ : A physiologically based pharmacokinetic analysis of capecitabine, a triple prodrug of 5-FU, in humans, the mechanism for tumor-selective accumulation of 5-FU. Pharm Res 200I, 18(8): II90-1202.

5. Vossen M, Sevestre M, Niederalt C, Jang IJ, Willmann S, Edginton AN: Dynamically simulating the interaction of midazolam and the CYP3A4 inhibitor itraconazole using individual coupled whole-body physiologically-based pharmacokinetic (WBPBPK) models. Theoretical Biology and Medical Modelling 2007, 4(13): 13

6. Arikuma T, Yoshikawa S, Watanabe K, Matsumura K, Konagaya A: Ontology-Driven Hypothetic Assertion (OHA) for Drug InteractionPrediction. In Second International Multi-Symposiums on Computer and Computational Sciences (IMSCCS 2007) Edited by: Ni J, Cremer J, Seidel E, Dongarra J, Casavant TL, Huang S, Gu G. IEEE; 2007:I-8.

7. Yoshikawa S, Kenji S, Konagaya A: Drug interaction ontology (DIO) for inferences of possible drug-drug interactions. Medinfo 2004, I I:454-458.

8. Berners-Lee T, Hendler J: The Semantic Web. Nature 200I, 410(6832): 1023-1024.

9. Grenon P, Smith B: SNAP and SPAN: Towards Dynamic Spatial Ontology. Spatial Cognition and Computation 2004, I:69-103.

10. Grenon P, Smith B, Goldberg L: Biodynamic Ontology:Applying BFO in the Biomedical Domain. In Ontologies in Medicine Amsterdam: IOS Press; 2004:20-38.

II. Kanehisa M, Goto S: KEGG: Kyoto Encyclopedia of Genes and Genomes. Nucleic Acids Research 2000, 28:27-30.

12. Azuma R, Umetsu R, Ohki S, Konishi F, Yoshikawa S, Konagaya A, Matsumura K: Discovering dynamic characteristics of biochemical pathways using geometric patterns among parameter-parameter dependencies in differential equations. New Generation Computing 2007, 25:425-44I.

13. Konagaya A, Konishi F, Hatakeyama M, Satou K: The superstructure toward Open Bioinformatics Grid. New Generation Computing 2004, 22:167-176.

14. Konagaya A: Trends in life science grid: from computing grid to knowledge grid. BMC Bioinformatics 2006, 7(Suppl 5):SIO.

15. Konagaya A, Satou K, Eds: Grid Computing in Life Science: First International Workshop on Life Science Grid, LSGRID2004 Splinger; 2005.

16. Tan TW, Arzberger P, Konagaya A, Eds: Grid Computing in Life Sciences : Proceedings of the 2nd International Life Science Grid Workshop, LSGRID 2005 World Scientific Pub; 2006.

17. Mathijssen RHJ, van Alphen RJ, Verweij J, Loos WJ, Nooter K, Stoter G, Sparreboom A: Clinical Pharmacokinetics and Metabolism of Irinotecan (CPT-II). Clinical Cancer Research 200I, 7:2182-2194.

18. Slatter JG, Schaaf LI, Sams JP, Feenstra KL, Johnson MG, Bombardt PA, Cathcart KS, Verburg MT, Pearson LK, Compton LD, Miller LL, Baker DS, Pesheck CV, Raymond S, Lord I: Pharmacokinetics, Metabolism, and Excretion of Irinotecan (CPT- I I) Following I.V. Infusion of [1 4C]CPT-II in Cancer Patients. Drug Metabolism and Disposition 2000, 28(4):423-433.

19. Sai K, Saeki M, Saito Y, Ozawa S, Katori N, Jinno H, Hasegawa R, Kaniwa N, ichi Sawada J, Kazuo Komamura KU, Kamakura S, Kitakaze M, Yutaka Kitamura NK, Minami H, Ohtsu A, Shirao K, Yoshida T, Saijo N: UGTIAI Haplotypes Associated with Reduced Glucuronidation and Increased Serum Bilirubin in Irinotecanadministered Japanese Patients with Cancer. Clin Pharmacol Ther 2004, 75:50I-5I5.

20. Ando Y, Saka H, Ando M, Sawa T, Muro K, Ueoka H, Yokoyama A, Saitoh S, Shimokata K, Hasegawa Y: Polymorphisms of UDP-Glucuronosyltransferase Gene and Irinotecan Toxicity: A Pharmacogenetic Analysis. Cancer Research 2000, 60:692 I-6926. 
21. Whitehouse LW, Information C, Menzies A, Dawson B, Cyr TD, By AW, Black DB, Zamecnik J: Mouse hepatic metabolites of ketoconazole: Isolation and structure elucidation. J Pharm Biomed Anal 1994, I 2(I I): |425- |44|.

22. Heel R, Brogden R, A Carmine PM, Speight T, Avery G: Ketoconazole: A review of its Therapeutic Efficacy in Superficial and Systemic Funfal Infections. Drugs 1982, 23: I-36.

23. Kehrer DF, Mathijssen RH, Verweij J, de Bruijn P, Sparreboom A: Modulation of Irinotecan Metabolism by Ketoconazole. Journal of Clinical Oncology 2002, 20( I 4):3 I22-3 I 29.

24. OWL Web Ontology Language Reference [http:// www.w3.org/tr/owl-ref/]

25. Haaz MC, Riche C, Rivory LP, Robert J: Biosynthesis of an aminopiperidino metabolite of irinotecan [7-ethyl-10-[4-(I-piperidino)-I-piperidino]carbonyloxycamptothecine] by human hepatic microsomes. Drug Metabolism and Disposition 1998, 26(8):769-774.

26. Haaz MC, Rivory L, Rich C, Vernillet L, Robert J: Metabolism of Irinotecan (CPT-II) by Human Hepatic Microsomes: Participation of Cytochrome P-450 3A and Drug Interactions. Cancer Research 1998, 58(3):468-472.

27. Slatter JG, Su P, Sams JP, Schaaf LJ, Wienkers LC: Bioactivation of the Anticancer Agent CPT-II to SN-38 by Human Hepatic Microsomal Carboxylesterases and the in Vitro Assessment of Potential Drug Interactions. Drug Metab Dispos 1997, 25(I0): I I57-I I64.

28. Gagne JF, Montminy V, Belanger P, Journault K, Gaucher G, Guillemette C: Common human UGTIA polymorphisms and the altered metabolism of irinotecan active metabolite 7-ethylI 0-hydroxycamptothecin (SN-38). Molecular pharmacology 2002, 62(3):608-617.

29. Chien JY, Lucksiri A, Charles S, Ernest I, Gorski JC, Wrighton SA, Hall SD: Stochastic prediction of CYP3A-mediated inhibition of midazolam clearance by ketoconazole. Drug Metab Dispos 2006, 34(7): $1208-1219$

30. Willmann S, Hohn K, Edginton A, Sevestre M, Solodenko J, Weiss W, Lippert J, Schmitt W: Development of a physiology-based whole-body population model for assessing the influence of individual variability on the pharmacokinetics of drugs. J Pharmacokinet Pharmacodyn 2007, 34(3):40I-43I.

31. Noy N, Fergerson R, Musen M: The knowledge model of Protege-2000: Combining interoperability and exibility. Proc EKAW 20002000.

32. Bomgaars L, Berg SL, Blaney SM: The Development of Camptothecin Analogs in Childhood Cancers. The Oncologist 200I, 6:506-516

33. Daneshmend TK, Warnock DW: Clinical Pharmacokinetics of Ketoconazole. Clinical Pharmacokinetics 1988, 14:13-34.

34. Rodriguez RJ, Acosta DJ: N-Deacetyl ketoconazole-induced hepatotoxicity in a primary culture system of rat hepatocytes. Toxicology 1997, I I7:123-131.

35. Rodriguez RJ, Proteau PJ, Marquez BL, Hetherington CL, Buckholz CJ, O'connell KL: Flavin-containing Monooxygenase-mediated Metabolism of $\mathrm{N}$-Deacetyl Ketoconazole by Rat Hepatic Microsomes. Drug Metab Dispos 1999, 27(8):880-886.

36. Tukey RH, Strassburg CP, Mackenzie PI: Pharmacogenomics of Human UDP-Glucuronosyltransferases and Irinotecan Toxicity. Molecular Pharmacology 2002, 62(3):446-450.

37. Garcia-Carbonero R, Supko JG: Current Perspectives on the Clinical Experience, Pharmacology, and Continued Development of the Camptothecins. Clinical Cancer Research 2002. 8:64I-66I.

38. Nozawa T, Minami H, Sugiura S, Tsuji A, Tamai I: Role of organic anion transporter OATPIB I (OATP-C) in hepatic uptake of irinotecan and its active metabolite, 7-ethyl-10-hydroxycamptothecin: in vitro evidence and effect of single nucleotide polymorphisms. Drug metabolism and disposition: the biological fate of chemicals 2004, 33(3):434-439.

39. Bodenreider O: The Unified Medical Language System (UMLS): integrating biomedical terminology. Nucleic Acids Research 2004, 32:D267-D270.

40. Rosse C, Mejino JLV: A reference ontology for biomedical informatics: the Foundational Model of Anatomy. Journal of Biomedical Informatics 2003, 36:478-500.
4I. Golbreich C, Zhang S, Bodenreider O: The Foundational Model of Anatomy in OWL: Experience and Perspectives. Journal of Web Semantics 2006, 4:18I-195.

42. Smith B, Ceusters W, Klagges B, Kohler J, Kumar A, Lomax J, Mungal C, Neuhaus F, Rector AL, Rosse C: Relations in biomedical ontologies. Genome Biology 2005, 6:R46.

43. Wielemaker J: SWI-Prolog 5.6 reference Manual, Updated for version 5.6.32. Amsterdam 2007.

44. Gansner ER, North SC: An open graph visualization system and its applications to software engineering. Software - Practice and Experience 2000, 30(II): 1203-1233.

45. Combes O, Barre J, Duche JC, Vernillet L, Archimbaud Y, Marietta MP, Tillement JP, Urien S: In vitro binding and partitioning of irinotecan (CPT-II) and its metabolite, SN-38, in human blood. Investigational New Drugs 2000, I 8: I-5.
Publish with Biomed Central and every scientist can read your work free of charge

"BioMed Central will be the most significant development for disseminating the results of biomedical research in our lifetime. "

Sir Paul Nurse, Cancer Research UK

Your research papers will be:

- available free of charge to the entire biomedical community

- peer reviewed and published immediately upon acceptance

- cited in PubMed and archived on PubMed Central

- yours - you keep the copyright 